\title{
Die nachweisbaren Strukturen der Federn von Archaeopteryx mit Anmerkungen zu Longisquama und diversen Proavis-Modellen
}

\author{
Burkhard Stephan ${ }^{1}$
}

Mit 9 Abbildungen

\section{Zusammenfassung}

Die Abstammung der Vögel, die Entwicklung des Vogelfluges und die Evolution der Vogelfeder sind die zentralen Themen, die sehr kontrovers diskutiert werden und bei denen Archaeopteryx als Taxon oder als Modell für die evolutiven Prozesse eine wesentliche Bedeutung zukommt. Dass der erste Fund von Archaeopteryx eine echte Vogelfeder ist und die Abdrücke bei den Skelettfunden von echten Federn stammen, ist offensichtlich. Sie haben einen Schaft, eine Innen- und eine Außenfahne, gebildet von Federästen und Federstrahlen. Der Beschreibung der Konturfeder rezenter flugfähiger Vögel folgt die Beschreibung der Konturfeder von Archaeopteryx. Am Berliner Exemplar lässt sich nachweisen, dass die Fahnen der Archaeopteryx-Federn wie bei den rezenten flugfähigen Vögeln von Bogen- und Hakenstrahlen gebildet werden.

Schließlich wird auf die gegenwärtige Diskussion von Longisquama hinsichtlich der Evolution der Federn und der Abstammung der Vögel sowie auf geeignete Proavis-Modelle eingegangen. Archaeopteryx hatte bereits hochentwickelte Federn. Wenn die Hautderivate von Longisquama als nonavian feathers den Vogelfedern homolog sind, dann sind sie ein Beleg dafür, dass die Federn in der mittleren Trias entstanden sind. Ihre Flugtauglichkeit erhielten sie erst durch Funktionswechsel. Quadrupede Gleiter eignen sich nicht als Proavis-Modelle.

Schlüsselwörter: Vögel, Archaeopteryx, Longisquama, Federstruktur, Evolution der Federn, Abstammung der Vögel, Proavis.

\begin{abstract}
The evident structures of the feathers of Archaeopteryx with a statement about Longisquama and diverse models of Proavis.

The origin of birds, the beginning of the flight of birds and the evolution of feathers are the central themes, which are controversially discussed, separate as well as together. Because of this, Archaeopteryx is of fundamental importance as taxon and as a model for evolutionary processes. That the first fossil finding of Archaeopteryx is a genuine bird feather and the moulds near the skeleton came from genuine feathers is evident. They have a shaft (rachis), an inner and an outer vane, which are formed by barbs and barbules. The existence of distal and proximal barbules has been provided. It was necessary to test, whether proof of that could be furnished on the feather moulds.

The description of the contour feather of Archaeopteryx follows the description of the contour feather of modern birds, which are able to fly. On the specimen of Archaeopteryx deposited at the Museum für Naturkunde (Berlin) can be shown that the vanes of the feathers are formed by anchoring of distal and proximal barbules, of hook and bow barbules. Additionally, a discussion of Longisquama concerning the evolution of feathers, the origin of birds and the suitable model of Proavis are considered. If derivates of Longisquama as nonavian feathers were homologous to bird feathers, they are an evidence for origin of the feathers in the Middle Triassic. They got their airworthy usefulness by change of function. Quadrupede gliders are unsuitable as Proavis models.
\end{abstract}

Key words: Aves, Archaeopteryx, Longisquama, feather structure, evolution of feathers, origin of birds, Proavis.

\section{Einleitung}

Die Entdeckung der einzelnen Feder 1860 und des ersten Skeletts mit Abdrücken von Federn 1861, das zum Londoner Exemplar von $A r$ chaeopteryx wurde, hätten nicht für so viel Aufregung gesorgt, wäre die prinzipielle Übereinstimmung mit Federn der rezenten flugfähigen
Vögel nicht sofort so offenkundig gewesen (Meyer 1861a, b, 1862, Heller 1959, Wellnhofer 1988). Nach wie vor hat Archaeopteryx eine zentrale Bedeutung einerseits als Taxon und für die verwandtschaftlichen Beziehungen sowohl der Vögel als auch der frühen Vögel untereinander sowie andererseits als Modell für die Evolution und Stammesgeschichte der Vögel und als

\footnotetext{
1 Museum für Naturkunde der Humboldt-Universtität zu Berlin, Invalidenstr. 43, D-10115 Berlin, Germany. Erhalten November 2000, angenommen Juni 2003
} 
Symbol dieser objektiven Gegebenheiten insgesamt.

Die Abstammung der Vögel, die Entwicklung des Vogelfluges und die Evolution der Vogelfeder sind die drei oft miteinander verquickt diskutierten Themenkomplexe, zu denen eine Vielzahl von Hypothesen und Theorien entwickelt wurden (Stephan 1974, 1979, 1987, Charig 1985, Berichte über die Roundtable Discussion während des 4. Internationalen Treffens der Society of Avian Paleontology and Evolution von Wittmer, Goslow, Jr. und Chiappe in Olson 1999: 327-344). Seltsamerweise spielt die Ableitung der Federn aus Schuppen noch immer eine Rolle (ProavisModelle; Diskussion in Stephan 1987). In diese Diskussion wird nun Archaeopteryx selbst einbezogen. Die Behauptungen, die Federabdrücke seien Fälschungen, sind allerdings längst widerlegt (Rietschel 1985b, Wellnhofer 1988, 2002).

Offenbar genügt es aber nicht (mehr), die heute bekannte Feinstruktur der Vogelfedern logischerweise auch für die Federn von $A r$ chaeopteryx vorauszusetzen. Auf Anregung von W. Bock, New York, suchte ich am Berliner Exemplar nach Stellen, die das Vorhandensein von Bogen- und Hakenstrahlen selbst anhand von Federabdrücken belegen könnten, wie sie Martin (1991: fig. 9) von Archaeopteryx beschrieben und abgebildet hat. Anlass hierfür war ein offener Brief von A. D. Walker, Newcastle upon Tyne, U.K., an mehrere Kollegen. in dem er bezweifelt, dass die Federn von Archaeopteryx der Struktur der Federn rezenter Vögel entsprechen und für Archaeopteryx und die Evolution der Vögel zu weitreichenden Schlussfolgerungen gelangt. Dabei spielt der Vergleich mit Longisqua$m a$ eine Rolle. Von anderen Autoren werden die vergrößerten Hautderivate von Longisquama völlig anders interpretiert.

Bevor die Ergebnisse der Untersuchung des Berliner Exemplars dargelegt werden, wird für den Vergleich und das leichtere Verständnis der Zusammenhänge der Bau der Konturfeder rezenter flugfähiger Vögel beschrieben. AbschlieBend wird auf den Vergleich zwischen Archaeopterx und Longisquama, die Evolution der Federn und die Abstammung der Vögel sowie die Proavis-Modelle eingegangen.

\section{Beschreibung der Konturfedern rezenter flugfähiger Vögel}

Die Feder wächst aus einem in die Haut eingesenkten Follikel in einem Hautmantel, dem Blut- kiel, heran, der beim Öffnen der Federfahne abfällt. Der basale Teil des Federkiels, die Spule, Calamus, weist eine Kammerung auf, die mit Besonderheiten des Federwachstums zusammenhängt.

Der auf die Spule folgende Abschnitt des Federkiels, Scapus, ist der Federschaft, Rachis. Von ihm gehen beidseitig Federäste, Rami, und von diesen wiederum beidseitig Strahlen, Radii, ab. Äste und Strahlen bilden zusammen die Federfahne, Vexillum, die aus der Innenfahne und der Außenfahne besteht. Die zur Federspitze ausgerichteten und an ihrer Unterseite mit Haken ausgestatteten Hakenstrahlen, die distalen Strahlen, überkreuzen die zum Federrand ausgerichteten und mit einer Krempe, dem Bogen, ausgestatteten Bogenstrahlen, die proximalen Strahlen. Äste und Strahlen entspringen jeweils in einem spitzen Winkel von weniger als $60^{\circ}$, so dass $\mathrm{Ha}$ ken- und Bogenstrahlen in einem Winkel von unter $90^{\circ}$ aufeinander treffen. Offenbar begünstigt dies das Verankern und Gleiten der Haken in den Bögen. Die Fahne ist fest und elastisch zugleich. Das ist der Grundaufbau der Konturfedern (Abb. 1). Von diesem ist eine Vielfalt an Abänderungen möglich und realisert (s. Bergmann 1987, Berndt \& Meise 1959, Bd. 1: 23-28, Brown et al. 1988: 96-101, 108-197, Bub 1985, Busching 1997).

Bei den Federn des Großgefieders, zu dem die Hand- und Armschwingen sowie die Schwanzfedern gehören, unterscheiden sich Außen- und Innenfahne deutlich. Die Außenfahne der Schwanzfedern wird vom zentralen Paar nach außen immer schmaler und fester, analog die Außenfahne der Handschwingen, so dass die äußeren Handschwingen eine sehr schmale Außenfahne haben - am ausgeprägtesten die mitunter stark eingebuchteten Handschwingen. Dieser Form- und Strukturwandel ist flugtechnisch begründet.

Die benachbarten Schwungfedern und auch die benachbarten Schwanzfedern sind jeweils so angeordnet, dass diese Federn beim Ausbreiten der Flügel und Spreizen des Schwanzes zum Erreichen einer großen Fläche sowie beim $\mathrm{Zu}$ sammenlegen der Flügel und des Schwanzes auf ein raumsparendes Ausmaß übereinandergleiten. Im zusammengelegten Flügel und Schwanz ist von oben betrachtet - stets die Außenfahne sichtbar (bei den inneren Armschwingen und einer der beiden mittleren Schwanzfedern auch die Innenfahne). Die Federn bleiben auch im maximal gespreizten Flügel rsp. Schwanz an der Basis überlagert, so dass ihr Übereinandergleiten stets gewährleistet ist - auch beim fliegenden 


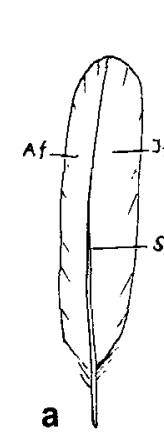

b

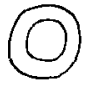
(0) 0

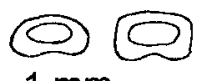
$1 \mathrm{~mm}$ c $100^{1}$ $\hat{4}$

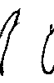

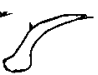
$1 \mathrm{~mm}$

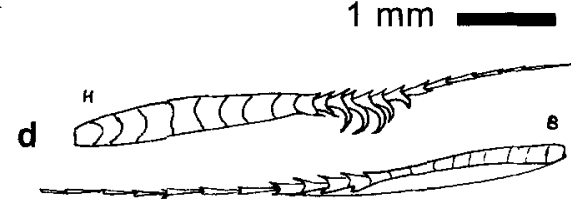

$0,1 \mathrm{~mm}$

Abb. 1. Strukturen der Konturfedern rezenter Vögel. a, Schema einer Feder. Af: Außenfahne; If: Innenfahne; S: Schaft. b, Varianten des Schaftquerschnitts. c, Varianten des Astquerschnitts. B Ansatz eines Bogenstrahls, H Ansatz eines Hakenstrahls. d, links Hakenstrahl, rechts Bogenstrahl. Fig. 1. Structure of contour feathers of modern birds. a, scheme of a feather. Af: outer vane, If: inner vane, S: shaft. b, variants of the shaft's cross-section. c, variants of the crosssection of the barbs. A: insertion of a bow (proximale) barbule, B: insertion of a hook (distal) barbule. d, left - hook barbule, right - bow barbule.

Vogel, der auf Änderungen von Luftströmungen und Windverhältnissen sofort reagieren muss.

Federn sind dreidimensionale Gebilde. Ihr Schaft ist im Querschnitt rund, oval, bohnenförmig oder auch fast quadratisch (Abb. 1). Auch die Äste haben einen wechselnden Querschnitt. Ihr basaler Teil ist mehr oder weniger oval, der dorsale Bereich kann zu einer Leiste verlängert und stark gebogen, der ventrale Teil spitz ausgezogen oder verbreitert sein (Abb. 1). Die Bogenstrahlen sind flach und an einem Rand aufgewölbt, die Hakenstrahlen haben Fortsätze nach ventral (einige zu Häkchen gekrümmt) und dorsal (Abb. 1). Die Bogenstrahlen entspringen am Ast tiefer als die Hakenstrahlen, die sich mit ih-

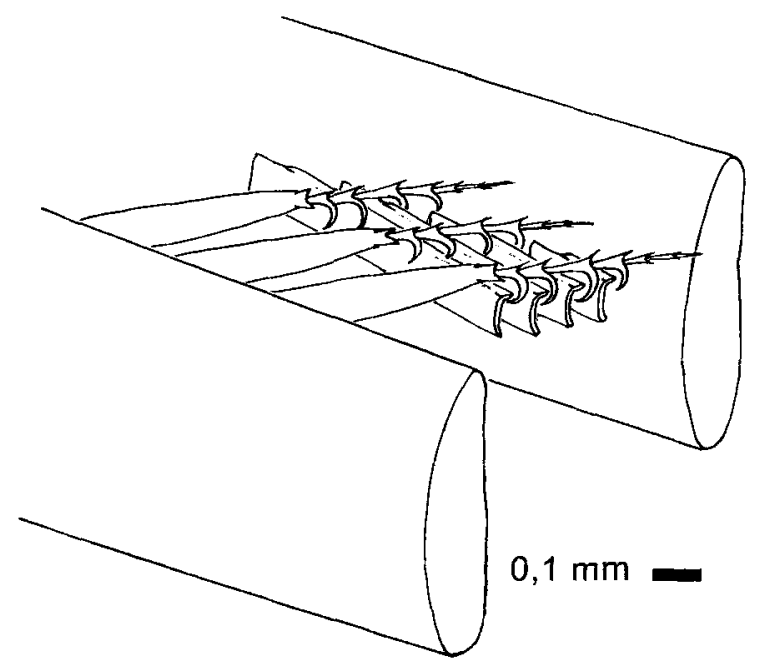

Abb. 2. Verzahnung der Haken- und Bogenstrahlen zur Bildung der Federfahne.

Fig. 2. Doveteiling of the hook and bow barbules for building a vane. ren Häkchen an der Krempe, dem Bogen, der Bogenstrahlen verankern (Abb. 2). Weitere Abbildungen der Verhakung bringen Brown et al. 1988, Bub 1985, Busching 1997, Griffiths 1996, Lucanus \& Stettenheim 1972 (letztere übernommen von anderen Autoren, u.a. Feduccia 1985). Beim Abdruck von Federn in einem Substrat können die im Innern der Fahnen verborgenen Strukturen - die Seiten von Schaft, Ästen und Strahlen, die nach ventral gerichteten Häkchen und die Bögen sowie die Verankerung zwischen Haken- und Bogenstrahlen - nicht abgebildet werden.

Betrachtet man eine Feder mit bloßem Auge, fallen der starke Schaft und die feinen und langen, sehr regelmäßig und dicht angeordneten Äste auf. Den Raum zwischen den Ästen füllen die Strahlen aus, von denen auf der Dorsalseite der Feder nur die Oberseite der Hakenstrahlen zu sehen ist (Abb 3), auf der Ventralseite der Feder nur die nach außen gerichtete Fläche der Bogenstrahlen. Bei Vergrößerung ist auch die jeweils andere Lage erkennbar. An Stellen, an denen die Verankerung zwischen Haken- und Bogenstrahlen gelöst ist, sowie am abgenutzten Federrand (Abb. 3, 4) sind die an den Ästen sitzenden Säume aus Haken- bzw. Bogenstrahlen zu erkennen. Die Fahne ist durch diese Struktur luftundurchlässig. So ist auch verständlich, dass auf einem viel gröberen Substrat, selbst dem

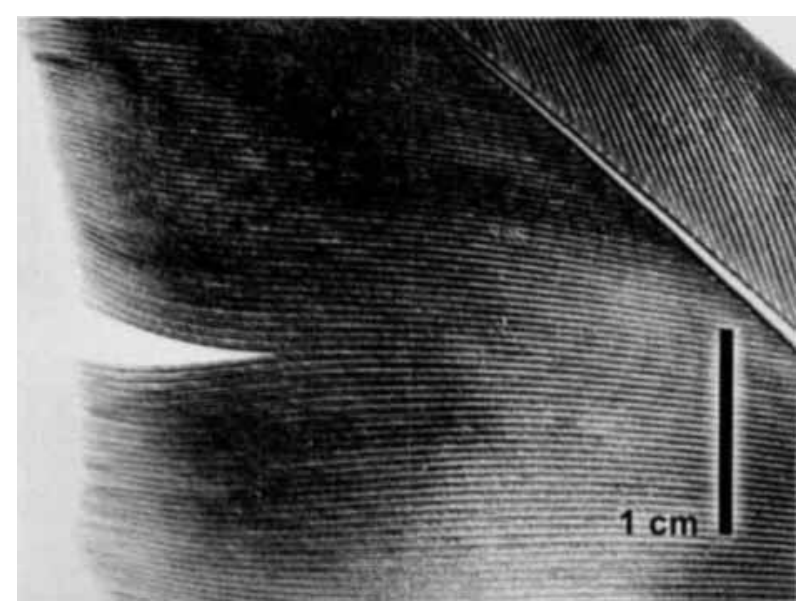

Abb. 3. Buteo buteo. Ausschnitt aus der Fahne einer Schwungfeder, von oben, mit Schaft, Ästen und - als Strichelung erkennbar - Hakenstrahlen (Fotos: W. Harre). An einer Stelle ist die intakte Fahne gestört. Hier sind außer den nahezu parallel zum Schaft ausgerichteten Hakenstrahlen die zum Federrand orientierten Bogenstrahlen als Saum zu erkennen.

Fig. 3. Buteo buteo. Part of a vane of a wingfeather, from dorsal, with shaft, barbs and - seen as broken lines - hook barbules. The intact vane is interrupted at one place. Here, besides the hook babules, which are almost parallel to the shaft, are also the bow barbules, which are directed to the border of the vane as a kind of hem. 


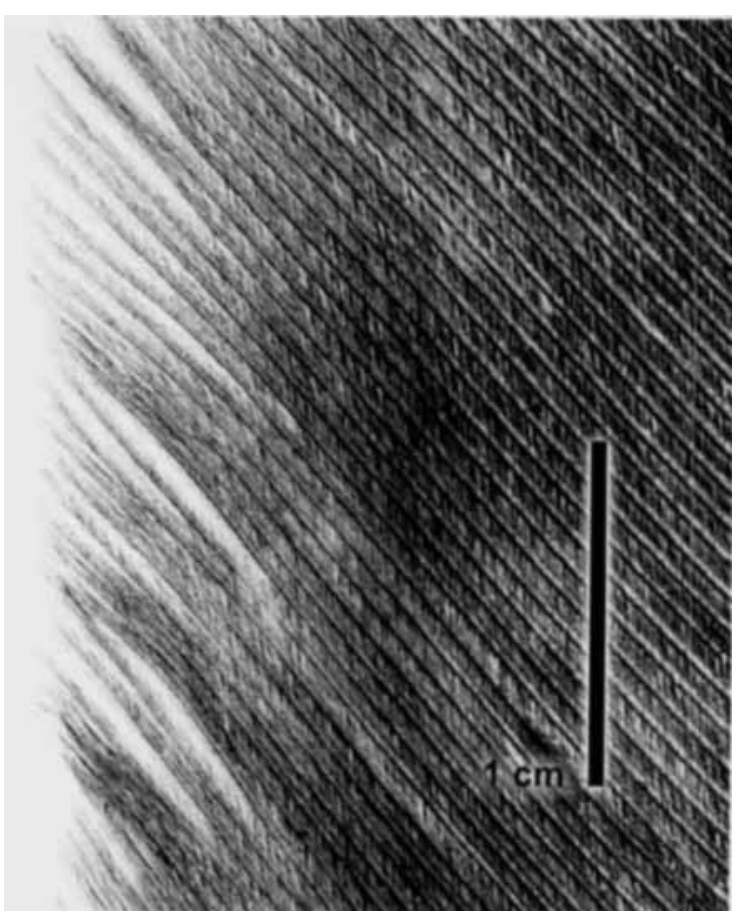

Abb. 4. Buteo buteo. Federfahne mit leicht zerschlissenem Rand. Äste mit je einem Saum aus Haken- und Bogenstrahlen. Fig. 4. Buteo buteo. Vane with slightly tattered edge. Barbs with a hem of hook barbules and bow barbules each. feinsten Schlamm, nur die jeweils äußere Fläche der genannten Strukturen einen Abdruck hinterlassen kann. Außerdem ist zu beachten, dass die ventralen Ränder der Äste verbreitert und die dorsalen Ränder verlängert und stark gekrümmt sein können, so dass die Äste in der Draufsicht breiter erscheinen, als sie sind (s. Querschnitt, Abb. 1).

\section{Die Struktur der Konturfedern von Archaeopteryx}

Die Federabdrücke des Berliner Exemplars (MB. Av. 100, MB. Av. 101) belegen eindeutig, dass die Federn eine Außen- und eine Innenfahne hatten, Strukturen, die nur durch Haken- und Bogenstrahlen möglich sind. Ferner ist zu erkennen, dass der Raum zwischen den Ästen ausgefüllt ist, ein Indiz dafür, dass Strahlen tatsächlich vorhanden waren (Abb. 5-8, s. auch Helms 1982, Rietschel 1985a: Tafeln 1 u. 2, Griffiths 1996, Martin \& Czerkas 2000).

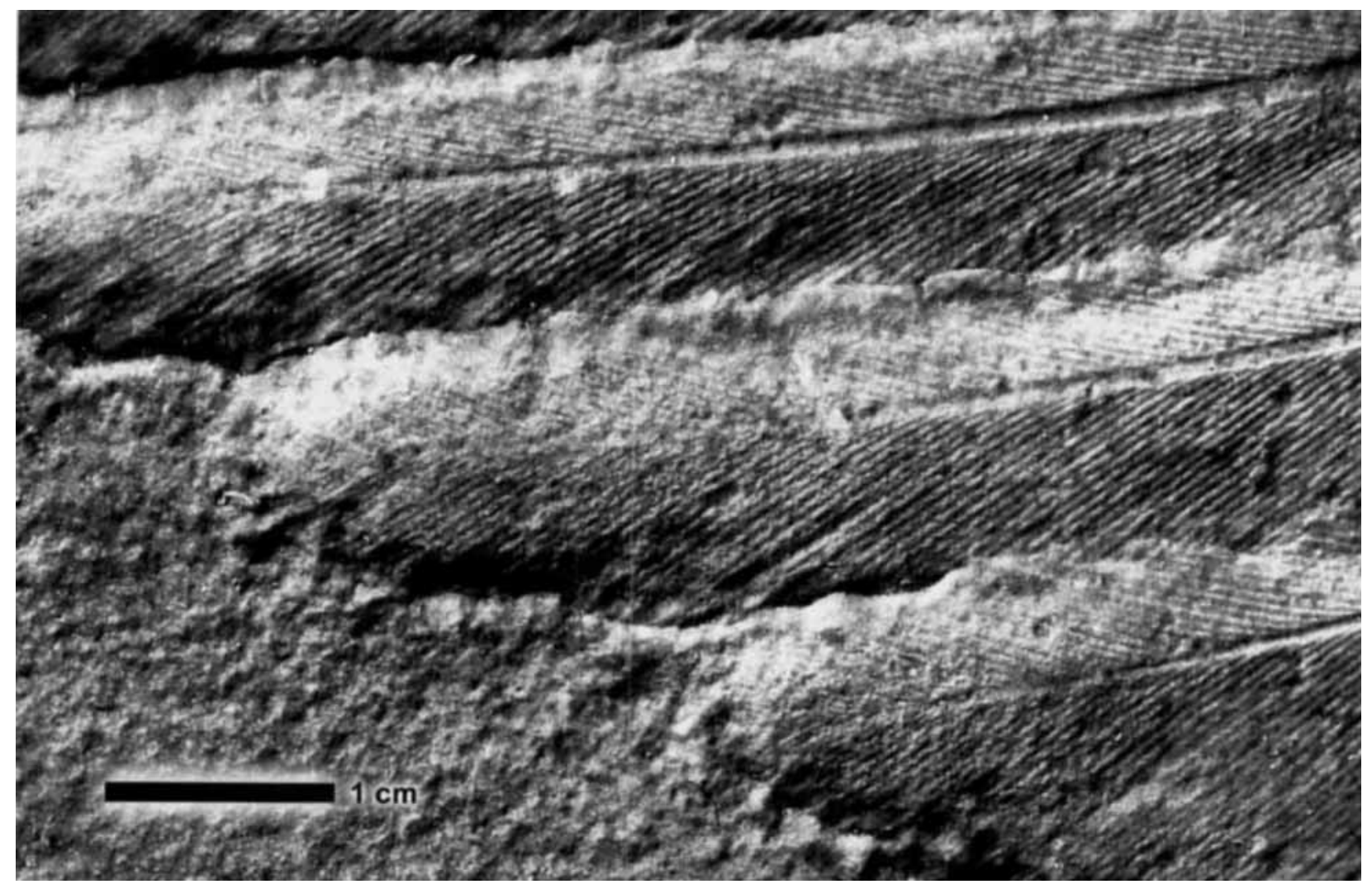

Abb. 5. Archaeopteryx lithographica (MB. Av. 101.1). Spitze des linken Flügels, 5.-7. Handschwinge (von außen gezählt). Deutlich erkennbar sind die Abdrücke von Schaft und Ästen. Der Rand der Außenfahne (s. vor allem dunkle Stellen) ist zerschlissen oder z.T. verwest. Kleinere Abschnitte sind in Dellen des Substrats gedrückt. Die Störung der einst geschlossenen Fahne ist an diesen Stellen längs der Äste erfolgt, d.h. durch Lösen der Verbindung zwischen Haken- und Bogenstrahlen. Fig. 5. Archaeopteryx lithographica (MB. Av. 101.1). Top of the left wing, 5th-7th primaries (counted inwardly). The moulds of the shafts and barbs are clearly seen. The edge of outer vane (see especially the darker places) is tottered or partly rotten. Little sections are pressed into dents. The disorder of the formerly closed vane at these parts always took place longitudinal the barbs. That is by loosing of the contact of hook and bow barbules. 


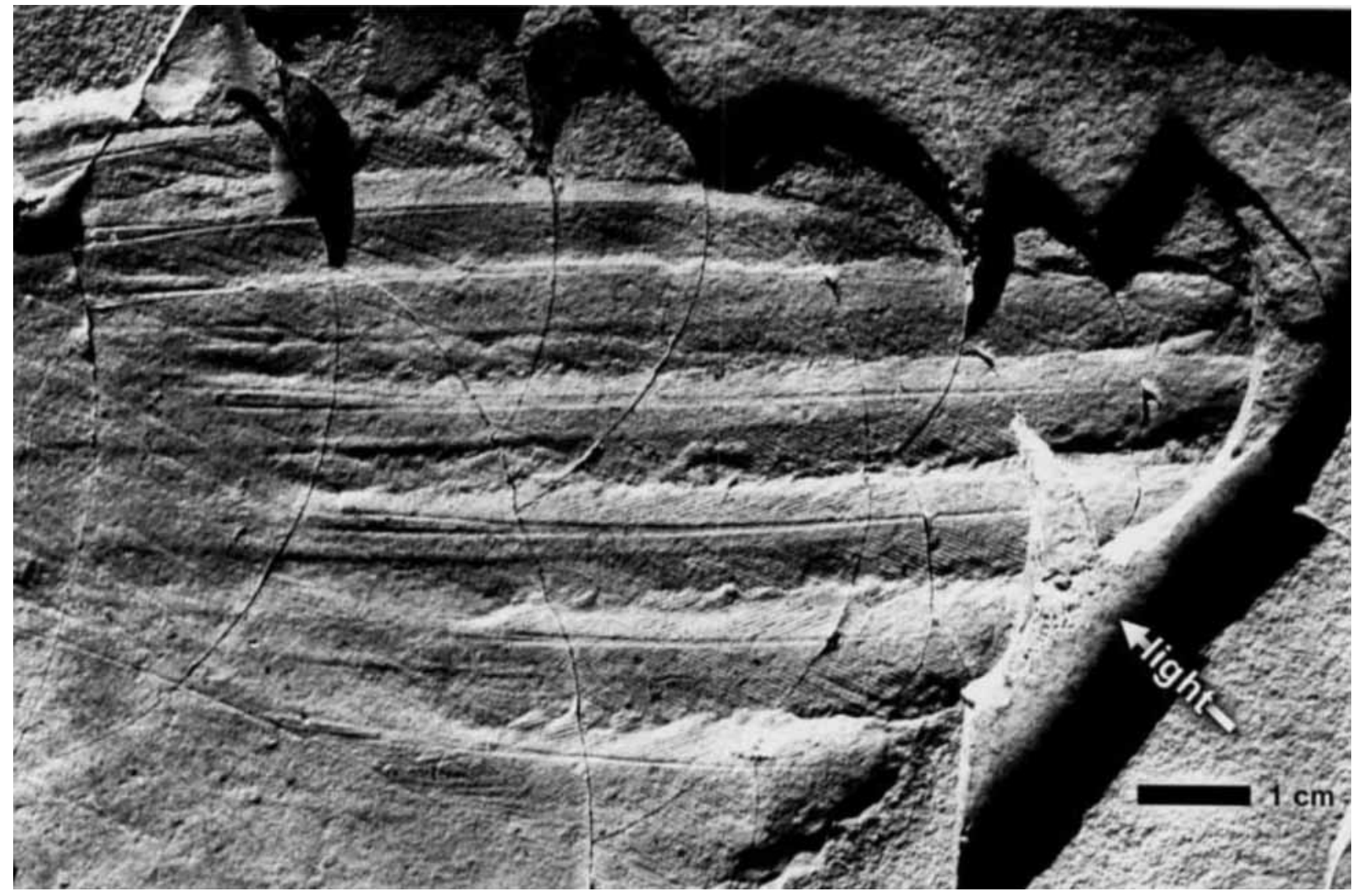

Abb. 6. Archaeopteryx lithographica (MB. Av. 101.2). Auch auf der Gegenplatte sind die Abdrücke von Schaft und Ästen der Flügel- und Schwanzfedern deutlich erkennbar. Rechts unten sind Schäfte und Fahnen von Flügeldeckfedern zu erkennen. Fig. 6. Archaeopteryx lithographica (MB. Av. 101.2). The counterpart shows clearly the marks of the shafts and barbs of the wing and tail feathers. Shafts and vanes of wing coverts at the bottom right are seen.

Außerdem gibt es an bestimmten Flügel- und Schwanzfedern des Berliner Exemplars Stellen, die nur durch das Vorhandensein von Hakenund Bogenstrahlen zu erklären sind. Wie von Federn rezenter Vögel bekannt ist, kann durch mechanische Einwirkung die Fahne der Feder aufgerissen werden, was bedeutet, dass an diesen Stellen die Bindung zwischen den Bogen- und Hakenstrahlen gelöst wurde (Abb 3.). Dieser Eingriff ist reversibel. Wir können die Haftung der Strahlen wieder herstellen, indem wir die Fahne zwischen Daumen- und Zeigefinger hindurchziehen. Die Vögel benutzen hierfür den Schnabel (beim „Putzen“ des Gefieders - man kann beobachten, wie sie einzelne Federn durch den Schnabel ziehen oder mit dem Schnabel im Gefieder stochern, womit sie nicht nur die Lage der Federn in Ordnung bringen sondern auch die Feinstruktur der Federfahnen).

Bei der Einbettung des Berliner Exemplars gab es auf dem Boden, auf dem der Vogel zu liegen kam, leichte Unebenheiten. Sie sind als Dellen erkennbar. Ihnen folgen Teile der Federfahnen, wodurch diese an diesen Stellen zerrissen (Abb. 5). Dieses Bild entspricht dem einer zerris- senen Stelle der Fahne (vgl. Abb. 3). An diesen Stellen sind die an den Ästen sitzenden Strahlen als Saum, der seitlich verschwommen ausläuft, auch auf dem Abdruck erkennbar. (Zur Einbettung von Archaeopteryx siehe Helms 1982.)

Analoge Stellen sind an alten abgenutzten Federn, vor allem kurz vor der Mauser, der Erneuerung des Gefieders respektive der einzelnen Federn, oder an bereits in Verwesung übergegangenen Federn erkennbar. Das trifft in geringem Maße auch auf die Ränder der Federn des Großgefieders des Berliner Exemplars zu, vor allem aber auf die einzelne Feder von Archaeopteryx, dem 1. Fund. Ihr Rand ist zerschlissen (Abb. 9). Bei dieser Feder ist der Schaft unterhalb der Fahne abgebrochen oder abgebissen, so dass der basale Teil des Kiels, die Spule, Calamus, fehlt. Wenn Greifvögel Federn erbeuteter Vögel rupfen, entsteht im Kiel ein Loch oder eine Kerbe, kleine Raubtiere unter den Säugetieren beißen den Kiel durch, große Raubtiere ziehen die Federn büschelweise heraus, wobei die Kiele abbrechen. Bei Mauserfedern bleibt der Kiel unbeschädigt erhalten (vgl. Brown et al. 1988: Abb. S. 75). 


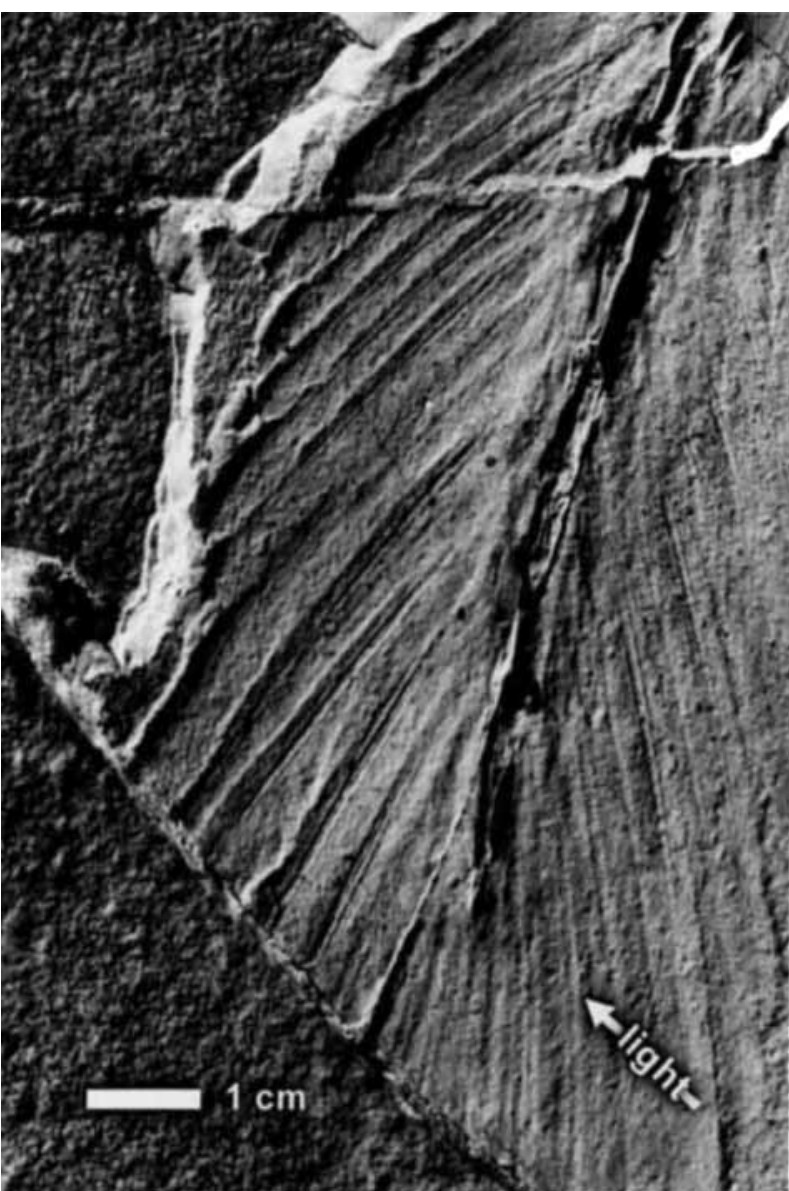

Abb. 7. Archaeopteryx lithographica (MB. Av. 101.1). Schwanz mit Abdrücken von Schwanzfedern. Schaft und Fahnen der Federn sind deutlich zu erkennen.

Fig. 7. Archaeopteryx lithographica (MB. Av. 101.1). Tail with marks of tailfeathers. Shaft and vanes of the feathers are clearly seen.

Die Federn des Großgefieders, die bereits erwähnten Hand- und Armschwingen sowie Schwanzfedern (Steuerfedern), und auch die Federn des Kleingefieders - das sind die Deckfedern von Flügeln und Schwanz, die Federn an Kopf, Hals, Rumpf und Beinen - bilden zusammen die Konturfedern. Allein schon aus den aerodynamischen Eigenschaften des Großgefieders ist zu schließen, dass die Urvögel ein Kleingefieder besaßen. Das Berliner Exemplar belegt dies durch die Abdrücke von Flügeldecken (Abb. 6) sowie Federn im Brustbereich (vor den Humeri), auf dem Rücken, am Schwanzansatz und an den Unterschenkeln. Letzteres ist in manchen Rekonstruktionen berücksichtigt, so von Pycraft (1910), Heilmann (1926, Gemälde), Burian in Augusta (1962), Reichel (1941) sowie auch beim Tetrapteryx-Stadium nach Beebe (Heilmann 1926), von anderen Autoren weniger oder gar nicht. Das übrige Kleingefieder ist immer dargestellt. Das Maxberger Exemplar (Heller

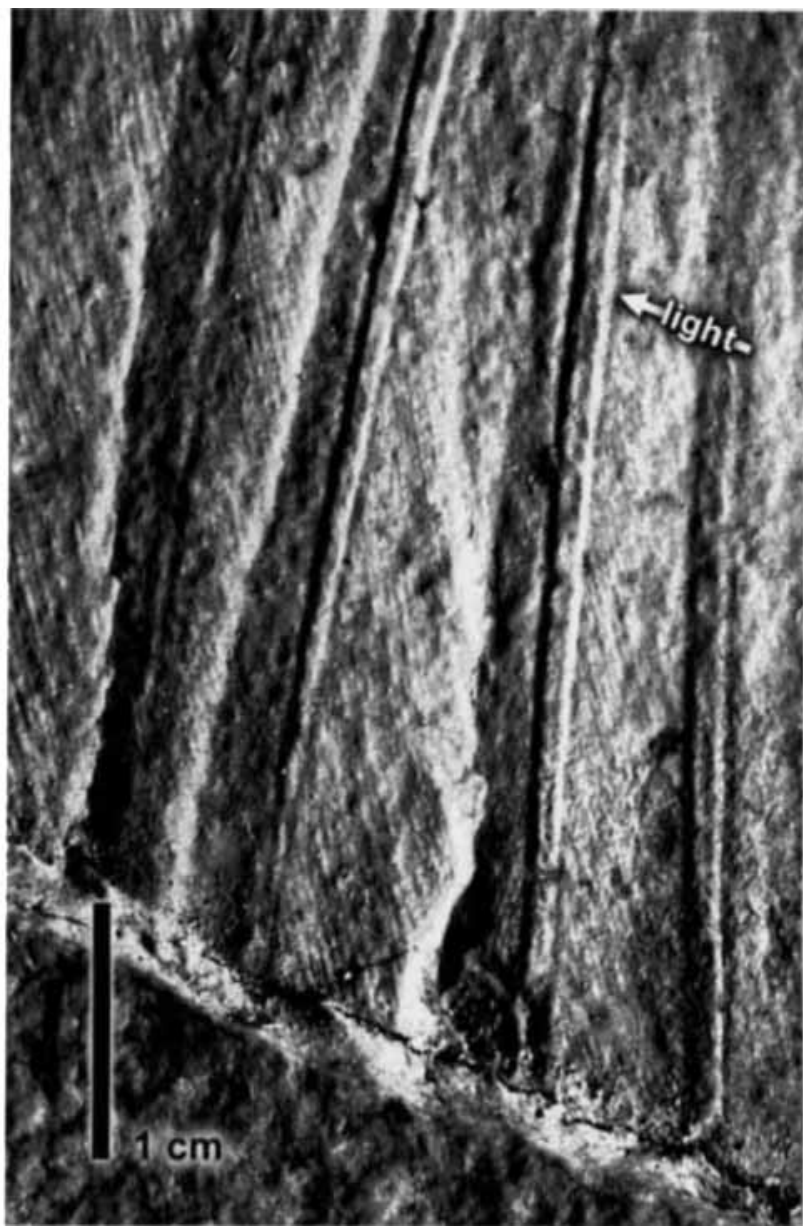

Abb. 8. Archaeopteryx lithographica (MB. Av. 101.2). Teil der Schwanzfedern (vergl. Abb. 7: linker unterer Bereich). Wie bei den Handschwingen erfolgte das Aufreißen der Fahnen längs der gerade verlaufenden Äste durch Lösen der Verankerung zwischen Haken- und Bogenstrahlen.

Fig. 8. Archaeopteryx lithographica (MB. Av. 101.2). Part of tailfeathers (see Figure 7: left lower sector). As with the primaries the burst of the vane took place longitudinal of the straight barbs by loosing the anchoring of the hook and bow barbules.

1959) zeigt ebenfalls deutlich Abdrücke einiger Federn des Kleingefieders. Auch für die anderen Exemplare von Archaeopteryx, für die alle $\mathrm{Fe}$ dern des Großgefieders nachgewiesen sind - bei den beiden letzten, dem Eichstätter und Solnhofener Exemplar bei schräger Beleuchtung im Streiflicht (Wellnhofer 1974, 1988) - war das Kleingefieder existenziell.

\section{Archaeopteryx, Longisquama, Proavis}

Wie aus obigem Vergleich hervorgeht, stimmen Form und Struktur der Federn von Archaeopter$y x$ und der Konturfedern rezenter flugfähiger Vögel prinzipiell überein. Zweifel hieran haben keine objektive Basis. 
Von einigen Autoren (Jones et al. 2000, Jones et al. 2001) ist Longisquama nun fest in die Überlegungen zur Evolution der Federn und zur Abstammung der Vögel integriert worden, was z. T. Rückwirkungen auf die Interpretation von Archaeopteryx hat, weswegen hier kurz darauf eingegangen werden soll.

Intern (Walker, s. Einleitung) wird eine Auffassung diskutiert, nach der zwar der erste Fund von Archaeopteryx lithographica, die mineralisierte einzelne Feder, als echte Vogelfeder anerkannt wird, die zu den Skeletten von Archaeopteryx gehörenden Abdrücke jedoch nicht von echten Federn stammen sollen. Dabei spielt u.a. der gute Erhaltungszustand der Fahnen, die nirgendwo aufgerissen, d.h. die Verhakungen von Hakenund Bogenstrahlen an keiner Stelle gelöst seien, eine Rolle. Die Fahnen der Federn von Archaeopteryx seien viel einfacher gebaut als die Federn rezenter Vögel: Die beiderseits des Schaftes vorhandenen Fahnen würden aus einer einheitlichen, regelmäßig gefalteten Lamina gebildet. Die „Federn" von Archaeopteryx seien essentiell den "Federn" von Longisquama ähnlich. Die Asymmetrie der Federn sei nicht nur beim aktiven Flug, sondern auch beim Gleiten notwendig. Unter Hinweis auf zahlreiche Skelettmerkmale (u.a. Schädel, Pubis, Hand) wird der Schluss gezogen, Archaeopteryx sei kein Vogel, sondern ein theropoder Dinosaurier und beide hätten nichts mit der Evolution der Vögel zu tun, die Vögel würden von frühen Crocodilomorphen abstammen. Das notwendige Übergangsstadium entspricht demjenigen der Baum-Theorie (quadrupedes Gleiten).

Mit dem Nachweis der Übereinstimmung der Federn von Archaeopteryx mit den Federn der Vögel (s. oben) entfällt auch die Schlussfolgerung zur Stellung von Archaeopteryx im System außerhalb der Aves.

In einem anderen Vergleich mit Longisquama gehen die Schlussfolgerungen in eine ganz andere Richtung. Feduccia (1985) hatte in den Haut- derivaten von Longisquama zunächst stark modifizierte verlängerte Schuppen gesehen und keinen Hinweis auf Ähnlichkeit mit Federn gefunden, weshalb sie für ihn als Übergang zwischen Reptilschuppe und Vogelfeder nicht in Betracht kamen. Er ist ebenfalls Anhänger der Baum-Theorie und betont die Entwicklung der Federn im Zusammenhang mit der Entwicklung des Fliegens. Die primäre aerodynamische Funktion der Vogelfeder werde durch die Asymmetrie der Fahnen der Handschwingen belegt. Sie ist auch die Begründung für die Flugfähigkeit von Archaeopteryx (Feduccia \& Tordoff 1979). ${ }^{2}$

Zusammen mit weiteren Autoren (Jones et al. 2000 ) ist Feduccia nun davon überzeugt, dass die Abdrücke der großen Hautderivate von Longisquama aufgrund der Struktur ihres Kiels der Nachweis der frühesten Federn sind. Ihr Basisteil hat einen Bau wie die Spule, Calamus, der Vogelfeder und steckt wie diese in einer Hauttasche. Diese Interpretation und das Primat der Flugfunktion der Federn ergibt die von den $\mathrm{Au}-$ toren vertretene Vorstellung, die verlängerten Hautderivate hätten dem Gleitflug gedient, wozu sie seitwärts abgespreizt werden mussten (Abb. siehe BBC News und Hawkes 2000). Das ist eine weitere Variante des quadrupeden ProavisStadiums.

Nach Sharov (1970), dem Entdecker von Longisquama insignis, handelt es sich um einen kleinen Pseudosuchier. Sein Rumpf war ca. 50, der Hals ca. 10, der Schädel ca $28 \mathrm{~mm}$ lang. Er hatte eine Furcula und eine lange schmale Scapula, Bauchrippen fehlen, die Vorderextremität hatte 5 Finger. Hinterkörper und Schwanz sind nicht erhalten. Die Hautderivate am Rumpf, die der Thermoregulation gedient haben könnten, sind 5-7 mal länger als breit und nicht so kompliziert gebaut wie die extrem verlängerten Gebilde auf dem Rücken. Diese sind etwa $120 \mathrm{~mm}$ lang, davon das verbreiterte Stück ca. $20 \mathrm{~mm}$. Der mittlere Abschnitt ist ca. $5 \mathrm{~mm}$ breit, das verbreiterte

\footnotetext{
2 Angemerkt sei hier, dass die Behauptung, die Handschwingen der Hühnervögel hätten eine wenig ausgeprägte Asymmetrie, nicht zutrifft (siehe z. B. Abb. in Brown et al. 1988). Ihr Flügel hat eine hohe Flächenbelastung, und sie müssen auch im windstillen Raum schnell vom Boden auffliegen können, um der Gefahr in letzter Minute zu entkommen. Ferner sei darauf hingewiesen, dass die Federn des Kleingefieders (Kopf-, Hals-, Rücken-, Brust- und Bauchfedern) symmetrisch gebaut sind (vgl. u. a. Abb. bei Brown et al. 1988, Busching 1997). Asymmetrisch sind vor allem die distalen Handschwingen und die äußeren Schwanzfedern, Federn, die während des Fluges besonders stark beansprucht werden. Die Asymmetrie dieser Federn entstand mit der Vervollkommnung des Fluges, nicht in seinen Anfängen.

Funktionswechsel von Strukturen war nicht selten. Bestimmte Strukturen entwickelten sich in Zusammenhang mit einer oder mehreren speziellen Funktionen. Sie müssen erst einen bestimmten Entwicklungsstand erreicht haben, bevor weitere Funktionen hinzukommen können. Werden letztere zur Hauptfunktion, können die bisherigen - im Falle der Federn Thermoregulation, Tarnung, beim Imponieren und bei der Balz - erhalten bleiben, wenn sie weiterhin im Leben der betreffenden Tiere eine wichtige Rolle spielen. Funktionswechsel der Federn bedeutet für die Evolution der Vögel u.a. die Kombination der Federn an Hand und Unterarm zu Tragflächen, d.h. Integration in eine neue funktionelle Einheit, die Flügel, als Handund Armschwingen, obere und untere Flügeldecken, Alulafedern. Die Federn des Kleingefieders bilden zusammen die Stromlinienform des fliegenden Vogels. Die Anordnung der Flügelfedern ist einmalig und ebenso wichtig wie die Federn selbst.
} 


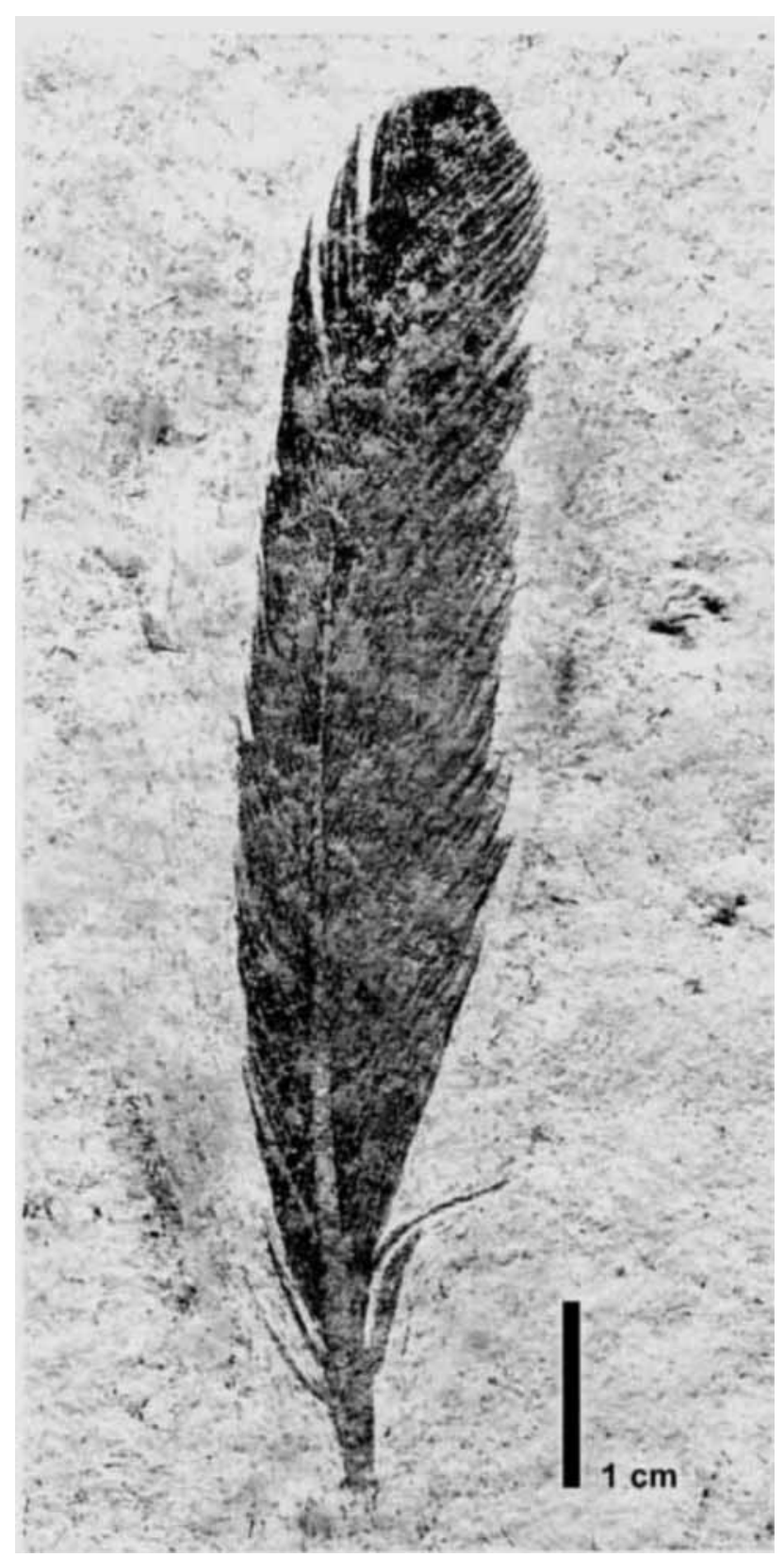

Abb. 9. Archaeopteryx lithographica. Einzelne Feder (MB. Av. 100). Die fossile Feder zeigt Übereinstimmung mit dem Bau einer Konturfeder rezenter Vögel - mit Schaft, schmaler Außen- und breiter Innenfahne. mit Ästen und z. T. abgenutztem Rand.

Fig. 9. Archaeopteryx lithographica. Solitary feather (MB. Av. 100). This fossil feather shows in correlation to feathers of modern birds the typical construction of a contour feather with shaft and narrow outer vane and broad inner vane. with barbs and partly disturbed edge.

Ende, die Fahne, etwa doppelt so viel. Deren Oberfläche ist geriffelt (s. Sharov 1970: Tafel VIII Bild 2). In der Mitte verläuft der Schaft. Der kompakte Saum der Fahne ist vorn viel breiter als der Schaft und hinten fast so breit wie dieser. Der Teil des Kiels, der der Spule der Feder homolog ist, reicht bis an die Fahne heran, ist also wesentlich länger als bei einer Vogelfeder. Sharov (1970) schreibt, dass diese Struktur im
Innern dieses Abschnittes an die Federseele der Spule einer Vogelfeder erinnert und offenbar dieselbe Funktion beim Wachstum hatte. Er nimmt an, dass diese Gebilde eine Fallschirmwirkung hatten und den Fall verlangsamten. Dabei waren sie schräg nach oben gestellt. Sharov kann zwar keinen Übergang von Longisquama zu den frühen Vögeln erkennen, hält es aber für möglich, dass die Vorfahren der Vögel solche Schuppen gehabt haben könnten, und Longisquama mit den Vogelvorfahren nah verwandt war. Longisquama lebte vor 230 Millionen Jahren (Unwin et al. 2000: mittlere bis obere Trias) in Mittelasien (Fergana-Becken im heutigen Kirgistan). Seine Stellung im System steht noch nicht fest.

Die langen Gebilde könnten auch eine ähnliche Funktion gehabt haben wie die Kehlfalte der Baumleguane (Norops) oder die Rückenkämme der Baumechsen, z. B. der Basilisken (Basilicus), und beim Imponieren und/oder Werben zur Geltung gebracht worden sein.

Jones et al. (2000) sehen in diesen langen Gebilden keine Schuppen, sondern nonavian feathers, und unterstreichen, dass - wenn diese Strukturen mit Vogelfedern homolog sind - die Entwicklung der Federn 75 Millionen Jahre vor der Existenz der voll befiederten Archaeopteryx erfolgte.

Letzteres würde erstens meine Überzeugung stützen, dass Federn nicht aus Reptilschuppen hervorgingen (Stephan 1974: 100, 1979: 112, 1987: 163, 168), was schon Lucanus \& Stettenheim (1972) - deren Arbeit mir erst vor wenigen Jahren zugänglich wurde - belegen, und zweitens auch die Erkenntnis, dass die Federn von Archaeopteryx bereits hoch entwickelt waren und die Vogelfedern deshalb wesentlich früher entstanden sein mußten.

Der Vergleich dieser nonavian feathers mit Vogelfedern zeigt, dass für eine Homologisierung nicht das gesamte Gebilde in Betracht kommt, sondern höchstens der basale Teil des Kieles mit den Luftkammern. Reisz \& Sues (2000) und Prum (2001) können keine Homologie zur Vogelfeder erkennen, und Unwin \& Benton (2001) betonen außerdem, dass nach bestimmten Skelettmerkmalen Longisquama kein Archosaurier ist.

Bei den quadrupeden Wirbeltieren, die gleiten wie z. B. die Flughörnchen, Pteromys, spannt sich eine doppelte Hautfalte an den Rumpfseiten zwischen Vorder- und Hinterextremität, bei den aktiv fliegenden Fledermäusen und Flughunden, Chiroptera, sowie den Flugsauriern, Pterosauria, sind/waren die Vorderextremitäten stärker einbezogen. Alle diese Tiere bleiben quadruped bei der Fortbewegung an Bäumen oder auf dem Boden. 
Ihre Flughaut wird durch die Körperwand und vor allem durch die Extremitäten gestützt. Die Hautderivarte von Longisquama saßen in Hauttaschen, an denen nur Hautmuskeln ansetzen. Diese reichen zum Aufrichten der Gebilde, nicht aber zu deren Stabilisierung bei horizontaler Stellung und hoher Beanspruchung während des Gleitfluges. Welche Muskeln, Sehnen, Bänder sollten die Stabilisierung bewirkt haben? Außerdem war die Wirbelsäule beweglich. Die Gebilde inserieren dorsal in der Nähe der Wirbelsäule und nicht an den Rumpfseiten, was - wenn überhaupt mit der Fortbewegung verbunden - für die Wirkung eines Fallschirms spricht, bei dem die langen Kiele als Streben fungierten. Manövrierfähig wäre ein solches Tier aber nicht.

Der quadrupede Gleiter gehört zu den frühesten Modellen von Proavis, dem hypothetischen Glied zwischen bestimmten Sauriern und Vögeln - s. Modelle von Pycraft (1910) und Steiner (1917) bis Tarsitano (1985) (Zusammenstellung in Stephan 1987). Charakteristisch sind Quadrupedie, ein Leben auf Bäumen (Baum-Theorie) und Gleitflug. Die Autoren lassen dabei die Federn aus verlängerten Reptilschuppen hervorgehen (obwohl Schuppen nur bei den Squamata auftreten, einer völlig anderen phylogenetischen Linie; s. hierzu auch Martin \& Czerkas 2000). Die Konstruktion der Hand von Archaeopteryx und den Vögeln bleibt unberücksichtigt, und die verlängerten Schuppen sind eher um den Ellenbogen gruppiert dargestellt als an der Hand (künftige Handschwingen) und am Unterarm (künftige Armschwingen).

Tetrapteryx-Vorstellungen eignen sich nicht als Proavis-Modelle, so auch diejenige in Form der Rekonstruktion von Microraptor gui durch $\mathrm{Xu}$ et al. (2003: 336) nicht. Sie geben kein „stage in bird evolution“ (p. 339) wieder. Darüber hinaus ist Microraptor gui in dieser Rekonstruktion mit Archaeopteryx-Flügeln ausgestattet. Entwicklungsstadien vor Archaeopteryx hatten jedoch noch keine so hoch entwickelten Flügel.

Die notwendigerweise bipeden Vorfahren der Vögel - bipede Archosaurier oder später bipede Theropoden - führten zur Boden-Baum-Theorie. Beide Theorien brauchen das Baumstadium für die Überwindung der Schwerkraft in den Anfängen des Fliegens. Seltsamerweise werden bei der zweiten Theorie aus bipeden Bodenbewohnern wieder quadrupede Baumbewohner bzw. solche, die auf den Bäumen ihre Vorderextremitäten zum Klettern und beim Gleiten einsetzen.

Boden-Theorien müssen für die Überwindung der Schwerkraft andere Varianten finden. Bei den meisten spielt die Verlängerung des Sprunges eine Rolle. Baum-Theorie und Boden-BaumTheorie erlauben hypothetisch eine allmähliche Vergrößerung der (Schuppen) Federn während der Evolution des Fliegens. Die Boden-Theorie setzt andere Funktionen wie z.B. die Thermoregulation für die anfängliche Evolution der Federn voraus (Übersicht und Diskussion s. Stephan 1987).

Bedenkt man, dass bei den rezenten Vögeln mehrere Typen von Federn - Konturfedern (s. oben, z. T. mit dunigen Strukturen), Pelzdunen, Borsten, Fadenfedern - und die Konturfedern in mannigfaltigen Formen und Größen sowie abgewandelt zu Schmuckfedern usw. vorkommen, so sollten weitere Strukturen, die aus Follikeln hervorgehen, nicht überraschen. Es müssen primär keine Federn oder Gebilde gewesen sein, die eine Fahne ausbildeten. Das Entstehen aus einem Follikel in einer Hauttasche weist nur auf die besondere Potenz der Haut dieser Tiere hin. Weitere Varianten solcher Hautderivate können durchaus existiert haben.

Aber nur eine Variante, nämlich diejenige, die Konturfedern am Unterarm und an der Hand sowie am Schwanz ausbildete, biped war und deren Vorderextremitäten frei waren von der Fortbewegung an oder auf dem Substrat, so dass sich Flügel mit dem für Vögel charakteristischen Gelenkautomatismus ausbilden konnten, kommt als Vorfahr der Vögel in Betracht, alle anderen nicht. Insofern entsprechen viele Proavis-Modelle und auch Longisquama - als Modell gedacht - nicht einem Bindeglied zwischen betreffenden Sauriern und den Vögeln.

Die langen Hautderivate von Longisquama deuten aber darauf hin, dass die Bildung von $\mathrm{Fe}$ dern in der mittleren Trias stattfand, wofür auch die relativ weit fortgeschrittene Entwicklung des Gefieders von Archaeopteryx spricht, und die Urvogelfedern das Ergebnis einer langen Evolution sind (Stephan 1974: 88, 1979: 99, 1987: 93). Dieser Schluss wird nun gestützt durch Hautderivate von theropoden Sauriern aus der Unterkreide Chinas, die mit den Borstenfedern der Vögel homologisiert werden (Cheng et al. 1998, $\mathrm{Xu}$ et al. 1999, 2000, 2001, Ji et al. 2001, Wellnhofer 2002) sowie dem Nachweis von tubulären borstenartigen Hautderivaten von Psittacosaurus, einem nicht-theropoden Saurier aus der Unterkreide Chinas (Mayr et al. 2002). Diese unverzweigten Gebilde haben Verbindung zu den Dornfortsätzen (Spinae dorsales) und Praezygapophysen der Schwanzwirbel. Der aus ihnen gebildete Schwanzborstenkamm hatte Signalfunk- 
tion. Bei den Vögeln dienen borstenartige $\mathrm{Fe}-$ dern als Wimpern dem Schutz der Augen sowie als Bart- oder Schnurrborsten am Schnabelwinkel als Fibrissen dem Tasten und als reusenartige Vergrößerung der Schnabelspalte beim nächtlichen Insektenfang. Die Borsten der theropoden und nicht-theropoden Saurier sind Grundlage für die Annahme, dass sich filamentartige Hautderivate vor der Evolution des Fliegens entwickelten (Xu et al. 1999, 2001, Brush 2001, Ji et al. 2001, Sues 2001, Wellhofer 2001, 2002, Mayr et al. 2002). Wellnhofer gibt das von $\mathrm{Xu}$ et al. entwickelte Modell der Evolution der Vogelfeder, d. h. einer Konturfeder, wieder und diskutiert weitere Funde. Das erste Stadium dieses Modells ist ein tubuläres zylindrisches faden- oder borstenartiges Gebilde, das dritte Stadium ist ein Gebilde aus einem längeren verzweigten Schaft, beim vierten Stadium sind auch die Äste verzweigt. Das zweite Stadium aber ist kein Übergangsstadium, denn es entspricht einer Pelzdune (Teleoptil): Viele Äste gehen von einem kurzen Schaft aus. Die Nestdune (Neoptil) ist eine dunige Struktur an der Spitze einer Konturfeder, die bei Ausbildung dieser Feder abfällt, sie hat eine strahlige Basis (ausführlich bei Brown et al. 1988, Busching 1997 u.a.). Pelzdunen stehen zwischen den Konturfedern und sind von diesen völlig bedeckt. Pelzdunen, Konturfedern, Fadenfedern und Borsten sind nicht-austauschbare Varianten des Gebildes Vogelfeder.

Es ist üblich geworden, Cladogramme zu erstellen. Mitunter sind es über ein Dutzend (s. Stokstad 2000). Sie helfen jedoch nicht weiter, weil die ausgewählten Merkmale unterschiedlich bewertet werden können. Die Lösung ist die Rekonstruktion des Werdens des gesamten Komplexes „Vogel“. Ein unter Beachtung der genannten Zusammenhänge entwickeltes ProavisModell zeigt Stephan (1987: 184-185).

Nach dem jetzigen Kenntnisstand sind für Proavis als Modell des Entwicklungsstadiums zwischen bipeden Sauriern und Vögeln folgende Details zu beachten:

- Klein, bis Drosselgröße

- Bipedie, Hinterextremitäten mit 4 Zehen, die erste nach hinten gewendet. Knie und Fersen gebeugt. Keine sekundäre Rückkehr zur Quadrupedie. Daher kein Gleitflug, sondern Beginn des Fliegens mit dem Fallschirmsprung (Vorderextremitäten nicht horizontal schräg nach vorn gestreckt, sondern leicht gewinkelt V-förmig nach oben gehalten). Bipede Lebensweise an Gebirgshängen und Flussterrassen, wo es immer ausreichende Luftbewegungen gibt.
- Verkürzung des Rumpfes und Näherung der Hüft- und Schultergelenke an den Körperschwerpunkt. Femora nach vorn-außen, Humeri nach hinten-außen gerichtet.

- Hals relativ lang und S-förmig gekrümmt.

- Schultergelenk bereits vor Proavis aus der Ebene des Brustbeins in Höhe der Wirbelsäule verlagert.

- Vorderextremitäten mit drei Fingern. Am Daumen entwickelten sich die Federn des Daumenfittichs, Alula, am langen Finger die distalen Handschwingen, am Metacarpus die inneren Handschwingen, an der Ulna die künftigen Armschwingen.

- Automatismus der Bewegung von Ellenbogenund Handgelenk, durch den beim Strecken des Ellbogengelenkes zugleich das Handgelenk gestreckt wird (bei Archaeopteryx ebenso entwickelt wie bei rezenten Vögeln).

- Von der Fortbewegung am Boden oder auf Bäumen völlig befreite Vorderextremitäten, da sich nur so aus ihnen Flügel entwickeln konnten. Die Verlängerung der Finger führte zur Vergrößerung der Fingerkrallen. Einen unmittelbaren funktionellen Bezug gibt es nicht.

- Reduktion des langen Schwanzes zusammen mit der Reduktion der Zähne und leichterer Bauweise.

- Entstehung der Vögel möglicherweise in einem Gebiet in Mittelasien.

\section{Danksagung}

Prof. Dr. W. Bock, New York, bat mich, am Berliner Exemplar von Archaeopteryx nach Indizien für den Feinbau der Federfahnen zu suchen. Frau W. Harre vom Institut für Paläontologie des Museums für Naturkunde der Humboldt-Universität zu Berlin machte die Foto-Aufnahmen. Dr. Unwin sowie Dr. S. Frahnert, Leiterin der Ornithologischen Abteilung des Instituts für Systematische Zoologie des Museums für Naturkunde Berlin, Dr. G. Mayr, Forschungsinstitut Senckenberg Frankfurt am Main, und Dr. G. Viohl, Jura-Museum Eichstätt, verdanke ich einige schwer zugängliche respektive neuere Literatur. Bei den englischen Texten half $\mathbf{H}$. Köhler, Wildau. Frau E. Siebert, Museum für Naturkunde, Berlin, hat die Abbildungen auf den Standard der Zeitschrift gebracht. Ihnen allen gilt mein aufrichtiger Dank.

\section{Literatur}

Augusta, J. 1962. Versteinerte Welt. 260 S., URANIA-Verlag, Leipzig, Jena, Berlin.

BBC News. 22. June, 2000. Earliest feathers fan controversy.

Bergmann, H.-H. 1987. Die Biologie des Vogels. 356 S., AULA-Verlag, Wiesbaden.

Berndt, R. \& Meise, W. 1959. Naturgeschichte der Vögel. Bd. 1, 390 S., Franck'sche Verlagshandlung, Stuttgart.

Brown, R., Ferguson, J., Lawrence, M. \& Lees, D. 1988. Federn, Spuren \& Zeichen der Vögel Europas - Ein Feldführer. 232 S., Gerstenberg Verlag, Hildesheim. 
Brush, A. H. 2001. The beginning of feathers. In Gauthier, J \& Gall, L. F. (eds). New Perspectives on the Origin and Early Evolution of Birds: 171-179. Yale Peabody Museum of Natural History, New Haven, N.J.

Bub, H. 1985. Kennzeichen und Mauser europäischer Singvögel. $211 \mathrm{~S}$., A. Ziemsen Verlag, Wittenberg Lutherstadt.

Busching, W.-D. 1997. Handbuch der Gefiederkunde europäischer Vögel. 400 S., AULA-Verlag, Wiesbaden.

Charig, A. J. 1985. Analysis of the Several Problems Associated with Archaeopteryx. In Hecht, M. K., Ostrom, J. H., Viohl, G. \& Wellnhofer, P. (eds). The Beginning of Birds: 21-30. Proceedings of the International Archaeopteryx Conference Eichstätt 1984. Eichstätt.

Cheng, P.-J., Dong, Z.-M. \& Zhen, S.-N. 1998. An exceptionally well preserved theropod dinosaur from the Yixian Formation of China. - Nature 391: 147-152.

Feduccia, A. 1985. On why the dinosaur lacked feathers. In Hecht, M. K., Ostrom, J. H., Viohl, G. \& Wellnhofer, P. (eds). The Beginning of Birds: 75-79. Proceedings of the International Archaeopteryx Conference Eichstätt 1984. Eichstätt.

Feduccia, A. \& Tordoff, H. B. 1979. Feathers of Archaeopteryx: Asymmetry vanes indicate aerodynamic function. Science 203: 1021-1022.

Griffiths, P. J. 1996. The Isolated Archaeopteryx Feather. Archaeopteryx 14: 1-26.

Hawkes, N. 2000. Birds 'take wing from lizard, nor dinosaurs'. - The Times, June 23.

Hecht, M. K., Ostrom, J. H., Viohl, G. \& Wellnhofer, P. (eds) 1985. The Beginning of Birds. 383 pp., Proceedings of the International Archaeopteryx Conference Eichstätt 1984. Eichstätt.

Heilmann, G. 1926. The Origin of Birds. 208 pp., H. F. \& G. Witherby, London.

Heller, F. 1959. Ein dritter Archaeopteryx- Fund aus dem Solnhofener Plattenkalken von Langenaltheim (Mfr.). Erlanger geologische Abhandlungen, Heft 31: 1-25.

Helms, J. 1982. Zur Fossilisation der Federn des Urvogels (Berliner Exemplar). - Wissenschaftliche Zeitschrift der Humboldt-Universität zu Berlin, Mathematisch-Naturwissenschaftliche Reihe 31: 185-198.

Ji, Q., Norell, M. A., Gao K.-Q., Ji, S.-A. \& Ren, D. 2001. The distribution of integumentary structures in a feathered dinosaur. - Nature 410: 1084-1088.

Jones, T. D., Ruben, J. A., Maderson, P. F. A. \& Martin, L. D. 2000. Longisquama fossil and feather morphology. - Science 291: 1901-1902.

Jones, T. D., Ruben, J. A., Martin, L. D., Kurochkin, E. N., Feduccia, A., Maderson, P. F. A., W. J. Hillenius, W. J., Geist, N. R. \& Alifanov, V. 2000. Nonavian feathers in a Late Triassic archosaur. - Science 288: 2202-2205.

Lucanus, A. M. \& Stettenheim, P. R. 1972. Avian Anatomy. Integument. Part I and II. Agriculture, Handbook 362. 750 pp., Agricultural Research Service, United States Department of Agriculture in Cooperation with Michigan Agricultural Experimental Station, Washington, D.C.

Martin, L. D. 1991. Mesozoic Birds and the Origin of Birds In Schultze, H.-P. \& Trueb. L. D. (eds). Origins of the Higher Groups of Tetrapods. Controversy and Consensus: 485-540. Cornwell University Press, Ithaca, New York.

Martin, L. D. \& Czerkas, S. A. 2000. The fossil record of feather evolution in the Mesozoic. - American Zoologist 40: 687-694.

Mayr, G., Peters, D. S., Plodowski, G. \& Vogel, O. 2002. Bristle-like integumentary structures at the tail of the horned dinosaur Psittacosaurus. - Naturwissenschaften 89: $361-365$.

Meyer, H. v. 1861a. Vogelfeder und Palpipes priscus von Solenhofen. - Neues Jahrbuch für Mineralogie, Geognosie, Geologie und Petrefakten-Kunde Stuttgart 1861: 561 (Briefwechsel an Professor Bronn).
- 1861b. Archaeopteryx lithographica (Vogel-Feder) und Pterodactylus von Solenhofen. - Neues Jahrbuch für Mineralogie, Geognosie, Geologie und Petrefakten-Kunde Stuttgart 1861: 678-679 (Briefwechsel, Mittheilungen an Professor Bronn).

- 1862. Archaeopteryx lithographica aus dem lithographischen Schiefer von Solenhofen. - Palaeontographica 10: $53-56$.

Olson, S. L. (ed.) 1999. Avian Paleontology at the Close of the 20th Century. Proceedings of the 4th International Meeting of the Society of Avian Paleontology and Evolution, Washington, D. C., 4-7 June 1996. - Smithsonian Contributions to Paleobiology, 89: 344 pp.

Prum, R. O. 2001. Longisquama fossil and feather morphology. - Science 291: 1900.

Pycraft, W. P. 1910. A History of Birds. 458 p., Methuen and Co. London.

Reisz, R. R. \& Sues, H.-D. 2000. The 'feathers' of Longisquama. - Nature 408: 428.

Reichel, M. 1941. L'Archaeopteryx. Un ancêtre des oiseaux. - Nos Oiseaux 16: 93-107.

Rietschel, S. 1985a. Feathers and wings of Archaeopteryx, and the question on her flight ability. In Hecht, M. K., Ostrom, J. H., Viohl, G. \& Wellnhofer, P. (eds). The Beginning of Birds: 251-260. Proceedings of the International Archaeopteryx Conference Eichstätt 1984. Eichstätt.

- 1985b. False forgery. In Hecht, M. K., Ostrom, J. H., Viohl, G. \& Wellnhofer, P. (eds). The Beginning of Birds: 371-376. Proceedings of the International Archaeopteryx Conference Eichstätt 1984. Eichstätt.

Sharov, A. G. 1970. A peculiar reptile from the Lower Triassic of Fergana. - Palaeontological Journal, Moscow 12: $127-130$.

Stephan, B. 1974. Urvögel Archaeopterygiformes. 167 S., A. Ziemsen-Verlag, Wittenberg Lutherstadt.

- 1979. Urvögel Archaeopterygiformes, 2. überarbeitete Auflage. 182 S., A. Ziemsen-Verlag, Wittenberg Lutherstadt.

- 1987. Urvögel Archaeopterygiformes, 3. überarbeitete Auflage. A. Ziemsen-Verlag, Wittenberg Lutherstadt, $216 \mathrm{~S}$.

Stokstad, E. 2000. Feathers, or Flight of Fancy? - Comments on Longisquama in Science. - New Focus, 25. June: 212.

Sues, H.-D. 2001. Ruffling feathers. - Nature 410: 1036-1037.

Unwin, D. M., Alifanov, V. R. Benton, M. J. 2000. Enigmatic small reptiles from the Middle-Late Triassic of Kirgizstan. In Benton, M. J., Shiskin, M. A., Unwin, D. M. \& Kurochkin, E. N. (eds). The Age of Dinosaurs in Russia and Mongolia: 177-186. Cambridge University Press, Cambridge.

Unwin, D. M. \& Benton, M. J. 2001. Longisquama fossil and feather morphology. - Science 291: 1899.

Wellnhofer, P. 1974. Das fünfte Skelettexemplar von Archaeopteryx. - Palaeontographica A 174: 169-216.

- 1988. Ein neues Exemplar von Archaeopteryx. - Archaeopteryx 6: $1-30$.

- 2001. Neues zum Ursprung der Vogelfeder. - Naturwissenschaftliche Rundschau 154: 366-367.

- 2002. Die befiederten Dinosaurier Chinas. - Naturwissenschaftliche Rundschau 155: 465-477.

Xu X, Tang Z.-L. \& Wang X.-L. 1999. A therizinosaurid with integumentary structures from China. - Nature 399: $350-354$.

Xu X., Zhou Z.-H. \& Wang X.-L. 2000. The smallest known non-avian theropod dinosaur. - Nature 408: 705-708.

Xu X., Zhou Z.-H. \& Prum, R. O. 2001. Branched integumental structures in Sinomithosaurus and the origin of feathers. - Nature 410: 200-204.

Xu X., Zhou Zu, Wang X, Kuang X., Zhang F. \& Du X. 2003. Four-winged dinosaurs from China. - Nature $\mathbf{4 2 1}$ : 335-340. 